pulseless. Restlessness was marked and from time to time he uttered feeble moans. He complained of sickness, faintmess, and a pain in the pit of his stomach. He appeared dreadfully anæmic, his tongue was dry, and his abdomen was distended with flatus and tender to the touch over the epigastrium. The recti resisted pressure by the tips of the fingers. Rest, ice to the epigastrium, and perchloride of iron in drop doses of the liquor sufficed to stop the hæmatemesis, but the following day brought a fresh complication, to wit, stercoraceous vomiting. Strange to say, this disagreeable innovation did not persist. Food by the mouth had been stopped from the first and this perhaps may account for the cessation of the vomiting, which only occurred during the morning of this day. The vomited material was very fotid.

At this stage of the attack I wrote to a well-known Liverpool surgeon asking for his advice with a view to operation. I. thought that, an opening having been made between the stomach and the colon, there would be very little likelihood of a satisfactory termination to the case unless that opening were closed.

The surgeon did not advise operation. He thought that considering the history the case was in all probability one of malignant ulceration and that the prognosis would be bad in any case. I therefore continued the rectal feeding and waited. Strange to say, there was no return of the fæcal vomiting after the first day.

After a fortnight of rectal feeding fluid nourishment was allowed by the mouth and gradually increased. The rectal feeding was discontinued. One of the most troublesome symptoms to deal with was insomnia All the ordinary hypnotics produced diagreeable effects and their useful effect did not last. Potassium iodide in five-grain doses thrice daily cured the insomnia. The patient was up and moving quietly about in a month from the onset of the illness. He was examined in June, 1899. The first attack has not been repeated, but warnings of its approach in the shape of digestive disturbances with excessive flatulence and tendermess over the epigastric region have recurred at intervals up to the present time. During these threatened attacks he has been kept in bed, dieted, and given iodide of potassium. Under this treatment the symptoms have passed off quickly. In appearance he is still cadaverous and anæmic. He says be feels very well. His height is $5 \mathrm{ft} .10 \frac{1}{2}$ in., and weight is 11 st. He has evidently not lost ground. The heart, lungs, and liver are normal. There is no skin rash, and no tenderness over the epigastrium on pressure. He sleeps fairly well. As to the past history, by occupation he was first a fireman and then a policeman. At a fire in 1883 he sustained an injury to his back which kept him in bed for two months in hospital. He states that he bas never had syphilis. Since the accident in 1883 he has never followed any occupation regularly, but has tried first one thing and then another. As to alcohol he has drunk a good deal of beer in his younger days, but for the last few years he has been very abstemious. I should add that the younger children present Hutchinson's signs of hereditary taint in their first teeth.

I am, Sirs, yours faithfully,

R. Lucrus Wood, M.B., Ch.B. Vict.

Knowsley, Lancashire, Sept. 20th, 1899

\section{"EPISTAXIS DUE TO A LEECH."}

\section{To the Editors of THE LANCET.}

SIRs,-Mr. Manasseh's case of epistaxis due to a leech emphasises the importance of a careful local examination in cases of hæmorrhage from the nose. Mr. Manasseh states that he has not found "that the leech is given as a cause of epistaxis in any of the text-books." In my book on "Diseases of the Nose and Throat" under the head of Epistaxis I say: "Leeches sometimes give rise to severe hæmorrhage." While I am pen in hand I should like to mention in connexion with the annotation in THE LANCET of Sept. 16th, p. 796, on the Treatment of Aneurysm by the Subcutaneous Injection of Gelatin that I tried this plan of treatment in a patient with aneurysm of the first part of the arch of the aorta but without any effect on consolidating the aneurysm. In spite of this failure $I$ had quite made up my mind even before reading your annotation to give the treatment another trial, as it is attended with but little risk or goain.

I am, Sirs, your faithfully, F. DE Havilland Hall.

\section{THE GAPS IN THE ROYAL ARMY MEDICAL CORPS. \\ To the Editors of THE LANOET.}

SiRs, - Truth on Sept."14th comments on the failure of the Royal Army Medical Corps to fill vacancies. At the last examination for 28 places only 14 were filled. Truth makes a reasonable suggestion-that candidates holding recognised diplomas should be only examined physically. It does seem strange why medical men who hold diplomas obtained only after the most strict and searching examination-examinations deemed sufficient by the General Medical Council for all other appointments-should be subjected to a further examination for commissions in the Army and Navy Medical Departments. Owing to a recent order of the General Medical Council practitioners are debarred from employing unqualified assistants. This has increased the demand for medical men in civil practice. If commissions in the Royal Army Medical Corps were granted after a strict physical examination and a three months' military training there would be little difficulty in selecting men of the very best physique for the Royal Army Medical Corps, which at the present time is nearly 100 short.

Sept. 20tb, 1899

I am, Sirs, yours faithfully,

EXCELSIOR.

\section{NOTES FROM INDIA.}

\section{(From our Special Correspondent.)}

The General Alarm of Plague and the Demand for the Prophylactic. - The Progress of the Epidemio.-The Escape of the Central Provinces.

THE spread of plague westward to Spain and Portugal seems to have excited more or less general alarm and hear that an unprecedented demand has suddenly arisen for the plague prophylactic fluid. The Government of India have been asked the cost of supplying from 50,000 to 100,000 doses and the earliest date at which this quantity could be despatched. It is also desired to know if in case of need 50,000 doses a week could be sent to London. Russia desires to obtain a considerable stock for Port Arthur. Italy has been making inquiries for home use and also Portugal in order to inoculate at Mozambique. The present laboratory is at Government House, Parel, Bombay, and has only recently been fitted up by the Government of India. About 10,000 doses a day can be turned out, but it is thought that still further enlargements will be required if the demand should increase beyond this amount.

The plague in India is still spreading, the returns for the week ending August 26th showing an increase of mortality to 3921 from 3585 in the previous week. To show the peculiar limitation of plague there were of this total no fewer than 3682 deaths in the Bombay Presidency, Poona City alone returning 1062 deaths. This frightful mortality in Poona has continued for the past three weeks and is at the remarkable rate of over 500 per thousand per annum. Several more Europeans have been attacked and I hear also that three nurses belonging to the Sassoon Hospital are down with enteric fever. Over 500 patients are in the General Plague Hospital. There are signs, however, that it is beginning to abate. Elsewhere throughout India little change is reported. Belgaum and Nasik districts and Kolapur state each return nearly 500 deaths. A few cases are reported from Bangalore City. There is no sign of diminution of plague in Calcutta, where about 40 cases are being reported weekly. There is, however, an ominous increase in the general mortality of the city of Bombay.

It is a remarkable circumstance that while plague has ravaged the adjoining districts of Bombay and the Deccan it has on each occasion of its appearance been apparently repelled from the territories of the Central Provinces. Small outbreaks occurred last November at two places and in one the disease was discovered on the occurrence of the very first case. Prompt and stringent measures were adopted. At the other place 19 cases had occurred when it was reported, but the quarter of the town was immediately cleared of all its inhabitants. Subsequently as cases continued to break out the whole town of 10,000 people was emptied. By March the disease was completely stamped ont. At two other places the same 